Revista General de Información y Documentación ISSN: 1132-1873

http://dx.doi.org/10.5209/rgid.70002

\title{
Tesis doctorales sobre museología en universidades españolas: un análisis bibliométrico, 1986-2018
}

\author{
Gonzalo Mochón-Bezares ${ }^{1}$; Ángela Sorli-Rojo ${ }^{2}$
}

Recibido: 6 de octubre de 2020 / Aceptado: 30 de noviembre de 2020

Resumen. El presente trabajo realiza un análisis bibliométrico de la producción de tesis doctorales sobre museología defendidas en universidades españolas entre los años 1986 y 2018, y recogidas en la base de datos TESEO del Ministerio de Educación y Formación Profesional. El número total de tesis recuperadas es 248 . Se han revisado la evolución de la producción de tesis en base a los años de defensa, los idiomas de redacción, las universidades de lectura, la temática y los doctores encargados de la dirección, así como la información sobre los miembros de los tribunales evaluadores. Se concluye que el número de tesis sobre museología se ha incrementado considerablemente en los últimos años, destacando la dispersión de la producción por universidades, una elevada concentración temática alrededor de la Historia del Arte y la Educación, una alta distribución entre los directores de tesis y alto grado de transitoriedad entre los miembros de los tribunales.

Palabras clave: Bibliometría; Tesis doctorales; Universidades; España; Museología; Producción científica

[en] Doctoral theses on museology in Spanish universities: a bibliometric analysis, 1986-2018

\begin{abstract}
The present work performs a bibliometric analysis of the production of doctoral theses on museology defended in Spanish universities between 1986 and 2018, and collected in the TESEO database of the Ministry of Education and Professional Training. The total number of theses recovered is 248 . The evolution of thesis production has been reviewed based on the years of defense, the writing languages, the reading universities, the subject and the doctors in charge of directing, as well as the information on the members of the evaluating courts. It is concluded that the number of theses on museology has increased considerably in recent years, highlighting the dispersion of production by universities, a high thematic concentration around the History of Art and Education, a high distribution among thesis directors and high degree of transience among members of the courts.
\end{abstract}

Keywords: Bibliometrics; Doctoral theses; Universities; Spain; Museology; Scientific production.

$1 \quad$ Documentalista

E-mail: gmochonb@gmail.com

2 Instituto de Ciencias de la Construcción Eduardo Torroja. CSIC

E-mail: angela.sorli@ietcc.csic.es 
Sumario. 1. Introducción. 2. Metodología. 3. Resultados 4. Conclusiones. 5. Referencias bibliográficas.

Cómo citar: Mochón Bezares, G.; Sorli Rojo, A. (2020). Tesis doctorales sobre museología en universidades españolas: un análisis bibliométrico, 1986-2018, en Revista General de Información y Documentación 30 (2), 339-355.

\section{Introducción}

Una tesis doctoral es una obra original de investigación que debe elaborar un doctorando para poder optar al grado académico de doctor, previa evaluación de dicha labor por parte de un tribunal de doctores expertos en la materia que se designa para la ocasión. Normalmente un trabajo académico de este tipo supone, por la extensión del documento que presenta el alumno para llegar a ser doctor, el esfuerzo y tiempo que este invierte en la realización del mismo, el primer gran estudio científico que realiza un investigador, y señala cuales podrían ser las líneas de investigación que se van a seguir en el futuro (Díaz Campo, 2014).

El análisis métrico de las tesis doctorales es una interesante vía para poder comprobar una dimensión de la actividad científica en las universidades y, de esta manera, poder evaluar su productividad en las diferentes disciplinas que en estas se imparten. Además, dicha forma de evaluación también resulta de gran utilidad para analizar la labor realizada por las otras personas implicadas en los procesos de elaboración (directores) y evaluación de las tesis doctorales (miembros de los tribunales), y las relaciones o vínculos que se han llegado a establecer entre esas personas (Repiso y otros, 2013). Para cada tesis doctoral juega un papel importante en su proceso de elaboración la figura del director, doctor experto en la materia sobre la que indaga el doctorando, al cual sirve de guía en su proceso de iniciación a la investigación y también como enlace con la comunidad científica y académica. La valoración académica de una tesis corresponde a un tribunal de doctores que hayan demostrado una capacidad investigadora suficiente, y de entre cuyos miembros destaca, de forma especial, la persona que lo preside.

La producción de tesis doctorales en las universidades españolas ha sido estudiada en numerosos trabajos, de los que muchos han sido considerados en el artículo debido a Fuentes Pujol y Argimbau Vivó (2010). En dicho trabajo se contabilizaron 36 artículos en los que se aborda el análisis métrico de tesis doctorales defendidas en universidades españolas, distribuyéndose el contenido de las tesis hasta en 18 disciplinas diferentes, entre las que destacan la Documentación, la Medicina y las Ciencias de la Educación por el número de trabajos que suman cada una de ellas.

Con posterioridad al estudio antes mencionado, las tesis que se han leído en las universidades españolas han continuado siendo objeto de atención para los investigadores y bibliómetras, especialmente las que se refieren a las disciplinas englobadas en las Ciencias de la Educación (Maz-Machado y otros, 2012; CurielMartín y Fernández-Cano, 2015; Moreno-Fernández y Moreno-Crespo, 2016; Sanchidrián Blanco, 2016; Ramos-Pardo y Sánchez-Antolín, 2017); las Ciencias de la Comunicación y la Publicidad (Repiso y otros, 2011a; Repiso Caballero y otros, 
2011; Marcos Recio y otros, 2012; Repiso y otros, 2013; Díaz Campo, 2014; Díaz Campo, 2015; Beladrón-Pazos y otros, 2019); la fotografía (Sánchez-Vigil y otros, 2014; Olivera Zaldua y otros, 2016) y las Ciencias de la Salud (Machan y Sendra Portero, 2018; Castello-Cogollos y otros, 2019; García Rubio y otros, 2019; SegarraSaavedra y Villena-Álarcón, 2020).

De acuerdo a lo señalado en el estudio de Fuentes y Argimbau (2010) y a lo observado en estudios posteriores sobre las tesis doctorales realizadas en España, se puede deducir que la principal fuente de información utilizada en los mismos ha sido la base de datos TESEO -recurso creado y editado por el Ministerio de Educación y Formación Profesional, en la cual se recogen datos de las tesis doctorales leídas en las universidades españolas desde 1976-, quedando otras bases de datos, como Dialnet o Tesis Doctorales en Red (TDR), y los repositorios de las universidades como fuentes secundarias con las que se han completado los datos incorrectos $\mathrm{u}$ omitidos en TESEO. Por otra parte, la estructura de análisis de los trabajos sobre tesis parece seguir en la mayoría de los casos un patrón común: revisión de la temática tratada en las tesis a través de los descriptores asignados por los propios doctorandos o personal de las universidades en TESEO, y el examen de varios aspectos sobre la producción de las mismas, tanto a nivel personal (directores y miembros de los tribunales), como institucional (universidades, departamentos universitarios u otras instituciones académicas). Sin embargo, solamente se han identificado diez artículos en los cuales los autores hayan empleado técnicas del análisis de redes sociales (ARS) para poner de manifiesto los vínculos que se han establecido entre los directores de las tesis y los miembros de los tribunales que las han evaluado: Delgado López-Cozar y otros, 2006; Casanueva Roche y otros, 2007; Repiso y otros, 2011; Repiso Caballero y otros, 2011; Maz-Machado y otros, 2012; Repiso y otros, 2013; Osca-Lluch y otros, 2013; Jiménez-Contreras y otros, 2014; Castelló-Cogollos y otros, 2019; Segarra-Saavedra y Villena-Álarcón, 2020.

A pesar de la diversidad de campos del conocimiento sobre los que se han publicado análisis de tesis leídas en España, hay materias que todavía no han sido tratadas, como es el caso de la museología. Esta disciplina se considera como la ciencia de los museos, sobre los que estudia diversos aspectos como su historia y rol en la sociedad; su tipología; el aspecto de su diseño arquitectónico; su organización y funcionamiento; la investigación y conservación de los objetos que exhiben, así como su presentación y formas de difusión al público (Weis, 1989). Esta definición demuestran que la museología, al abarcar el estudio de los variados aspectos que intervienen en la gestión de los museos, debe ser considerada una ciencia pluridisciplinar.

En lo que se refiere a estudios bibliométricos relativos a la museología que se han llevado a cabo en otros países, sólo se han encontrado sendos estudios acerca de la productividad científica de los doctorandos en universidades brasileñas. En el primer estudio, Bovolenta Ovigli (2015) analiza 31 tesis doctorales y 122 trabajos de máster presentados entre los años 1980 y 2010, que indagan sobre la educación en los museos de ciencias. Este autor constata que el incremento en la producción de dichos trabajos académicos durante el periodo estudiado está relacionado con la expansión de los programas de postgrado en educación científica que han sido implementados 
en las universidades brasileñas. El segundo artículo estudia 17 trabajos académicos (13 disertaciones y 4 tesis), recogidos en bases de datos sobre estudios de postgrado brasileñas, en los que se investiga la inserción de medios digitales interactivos, especialmente la realidad aumentada, en los espacios museísticos. Los autores concluyen que la cantidad de los trabajos académicos realizados resulta algo escasa y que ello es debido a que la citada temática no está todavía consolidada entre los grupos de investigación de ese país (Sardo Menezes y otros, 2019).

Una vez constatada la ausencia de análisis métricos relativos a tesis españolas sobre las disciplinas relativas a los museos, se decide realizar el presente estudio, cuyo objetivo principal es analizar distintos aspectos de la producción científica de tesis doctorales recogidas en la base de datos TESEO sobre museología entre los años 1986 y 2018, con el fin de evaluar la producción científica de las universidades españolas y el prestigio alcanzado por directores y evaluadores de tesis en base a la información recogida en la mencionada base de datos. El rango temporal de nuestro estudio comienza con la primera tesis recogida en TESEO que trata sobre museología (1986) y finaliza en 2018.

Tomando como referencia el objetivo principal, se han determinado los siguientes objetivos específicos:

- Analizar la evolución en la producción a lo largo de todo el periodo estudiado, tanto en lo que se refiere a las instituciones en las que defienden sus tesis los doctorandos como a los doctores encargados de la dirección de dichos trabajos académicos, para ver cuál es el rendimiento de las universidades y los directores en materia de investigación sobre museología.

- Identificar las diferentes temáticas abordadas en las investigaciones sobre museología para comprobar cuales son las líneas de investigación que se han establecido sobre dicha materia.

- Analizar la composición de los tribunales evaluadores de tesis con el fin de comprobar la importancia de cada uno de sus miembros y ver el grado de concentración en determinados profesores.

- Observar la diferencia que se establece entre los géneros de los doctorandos, los directores de las tesis y los miembros de los tribunales para ver la importancia que tiene cada sexo en los colectivos de producción, dirección y evaluación de los trabajos.

\section{Metodología}

Se realiza un análisis bibliométrico descriptivo y longitudinal de carácter retrospectivo sobre las tesis leídas en las universidades españolas que tratan algún aspecto de las disciplinas museología entre los años 1986 y 2018.

La fuente principal de información utilizada ha sido la base de datos TESEO (2020). La mencionada base de datos es el recurso más completo que existe en España en lo que se refiere a la información sobre las tesis doctorales, ya que incluye en la mayoría de los registros los nombres de las personas que han formado parte de los tribunales evaluadores de tesis, los cuales no son recogidos de forma sistemática 
en los registros de otras bases de datos, repositorios de universidades o catálogos de bibliotecas.

A pesar de que TESEO proporciona a los usuarios información que se considera de gran valor, dicha base de datos presenta una serie de deficiencias. Algunas de estas se han señalado en varios artículos y son debidas en gran parte a la falta de control en el ingreso de los datos, labor que realizan los doctorandos o personal de las propias universidades. Dichas deficiencias son las siguientes: su exhaustividad no es muy elevada, especialmente en lo que se refiere a su periodo más antiguo; presenta registros incompletos, en los que suelen faltar nombres de departamentos universitarios, directores de tesis y miembros de tribunales; su actualización es lenta; no existe normalización en los nombres de los directores o miembros de tribunales; se ha constatado asignación imprecisa de palabras clave del Tesauro UNESCO, sistema de clasificación usado en la base de datos TESEO; hay inexactitudes en las fechas de lectura de las tesis; la interfaz de consulta solamente está disponible en castellano; a veces se producen errores en el servidor o en la página web que impiden una navegación fluida. (Delgado López-Cozar y otros, 2006; Fuentes Pujol y Argimbau Vivó, 2010; Repiso Caballero y otros, 2011; Díaz-Campo, 2014; Díaz Campo, 2015). A esta lista de ausencias y deficiente funcionamiento se puede añadir la limitación que supone manejar los resultados obtenidos en las consultas, pues sólo se pueden ver los registros de uno en uno y no se ofrece la posibilidad de su descarga conjunta en ningún formato.

Se han empleado otras fuentes pero solamente con el fin de completar las lagunas existentes en TESEO o bien normalizar nombres de personas o instituciones que en algunos casos puntuales han resultado dudosos. Las mencionadas fuentes son las siguientes: la base de datos Dialnet Tesis, el repositorio cooperativo Tesis Doctorals en Xarxa (TDX), el repositorio E-prints Complutense, el repositorio DIGIBUG de la Universidad de Granada y el repositorio RiuNET de la Universitat Politécnica de Valencia, entre otras.

En un primer momento de este estudio se buscó recuperar documentos en la base de datos TESEO mediante la consulta a través de los descriptores del tesauro UNESCO asignados a las fichas de las tesis por los propios doctorandos o los responsables en las universidades. El resultado obtenido por esta vía fueron 182 tesis que habían sido etiquetadas con el código 510106 Museología. Sin embargo, esta forma de consulta se rechazó posteriormente porque el descriptor museología sólo se utilizó en once registros entre los años 1976 y 2000, cifra que se antoja algo escasa. En una consulta posterior se pudo comprobar que un porcentaje significativo de los registros tenían mal asignado el descriptor, ya que su temática principal no era la museología o ciencia de los museos.

Al comienzo de la investigación se pensó que la consulta abarcara desde el año en que se creó la base de datos TESEO (1976) hasta 2018, pero posteriormente se decidió ajustarlo a 1986 como fecha de inicio por no haberse podido recuperar hasta dicho año ningún registro que se ajustara a la temática de la consulta.

Para evitar la limitación que supone la consulta por descriptores, se optó por otra vía en la que se empleó el término muse* en el título y/o el resumen, añadiéndole una restricción por tramos temporales para poder acceder a la información de todos 
los registros, porque TESEO sólo ofrece acceso a los 250 primeros resultados de cada consulta y su visualización debe hacerse de uno en uno. Empleando esta fórmula de búsqueda se obtuvo un total de 1577 referencias de tesis. Dichas referencias fueron revisadas por los autores del presente trabajo de investigación en base a los títulos de las tesis, sus resúmenes y los descriptores que se les habían asignado, considerando como válidas para nuestro estudio aquellas tesis que trataran alguno de los aspectos de la museología que se incluyen en las definiciones recogidas al principio de este apartado. El número final de documentos que se obtuvo tras esta selección fue de 248.

Se considera que la cantidad obtenida es un conjunto lo suficientemente representativo para comprobar las tendencias de las diferentes variables que son sometidas a evaluación en este estudio.

Para realizar el análisis bibliométrico de los documentos seleccionados se han analizado las siguientes variables:

1) Cifra de producción de tesis. Se toman los años del campo fecha de lectura de la base TESEO para analizar la evolución en la producción de tesis.

2) Idioma que se ha empleado en la redacción de cada tesis, con el objetivo de comprobar el grado de internacionalidad en lo que a la lengua se refiere.

3) Universidades de lectura de la tesis y su correspondiente departamento. Se busca comprobar la productividad de cada institución y de los departamentos en función de la disciplina que trata cada uno.

4) Temáticas tratadas en las tesis. Se agrupan los trabajos en base a los descriptores asignados en la base de datos a cada registro. Para la agrupación de los temas se usa como guía el Tesauro UNESCO, que es utilizado en TESEO para la indización de las tesis.

5) Directores de tesis, sobre los que se evalúa su productividad, el grado de colaboración que se da entre los directores (codirección) y la existencia de genealogías entre directores y doctorandos.

6) Miembros de los tribunales de evaluación, de los que se evalúa el grado de importancia de cada uno en base a la cantidad de tribunales a los que asiste.

En las variables de análisis que son relativas al estudio de personas (autores, directores y miembros de tribunales), se contemplan también los porcentajes por género para evaluar su presencia e importancia en cada uno de los tres grupos.

\section{Resultados}

\subsection{Resultados globales}

Las tesis sobre Museología leídas en universidades españolas entre 1986 y 2018 que están recogidas en la base de datos TESEO suman un total de 248. En algunos casos se han constatado las siguientes carencias en los registros: los nombres de los directores de las tesis no se incluyen en cuatro de ellos, fechados entre 1987 y 1990; los nombres de los departamentos universitarios a los que se vinculan las tesis no 
han sido recogidos en 34 registros, entre los que destacan los relativos a 13 tesis de la Universidad Complutense de Madrid; los miembros del tribunal no han sido recogidos en una ocasión.

La media anual de producción son 7,51 tesis por año y una producción al alza aunque con altibajos. Como se puede apreciar en la figura 1 , los resultados de los doce primeros años del periodo (de 1986 a 1997) resultan bastante escasos, no llegando a superar la cantidad de dos tesis leídas por año e incluso quedando algunas anualidades (1988 y 1993) sin documentos registrados. Durante el decenio siguiente (1998 a 2007) las tesis registradas por años mejoran ligeramente respecto al periodo anterior, si bien se aprecian descensos en algunos años. El último periodo (20082018) es con gran diferencia el más productivo, especialmente el trienio comprendido entre 2015 y 2017, que agrupa más de una tercera parte de la producción total. El descenso brusco en 2018 frente a los años anteriores puede deberse a cierta lentitud en el ingreso de datos y/o actualización de la base TESEO.

En lo que se refiere al género de los autores durante los treinta y tres años sometidos a estudio, el porcentaje de tesis producidas por mujeres ha resultado ser muy superior al de los hombres $(64,1 \%$ frente a $35,9 \%)$. Si se considera por separado la producción de cada uno de los periodos temporales señalados más arriba, se ve que la diferencia entre géneros es menor durante los once primeros once años $(58,8 \%$ para las mujeres y $41,2 \%$ para los hombres), pero al ir incrementándose el número de tesis registradas también aumentan los porcentajes de tesis que son debidas a mujeres mientras que descienden las realizadas por hombres: $66,6 \%$ frente a $33,3 \%$ entre 1998 y 2007, y 64,1\% frente a 35,9\% entre 2008 y 2018.

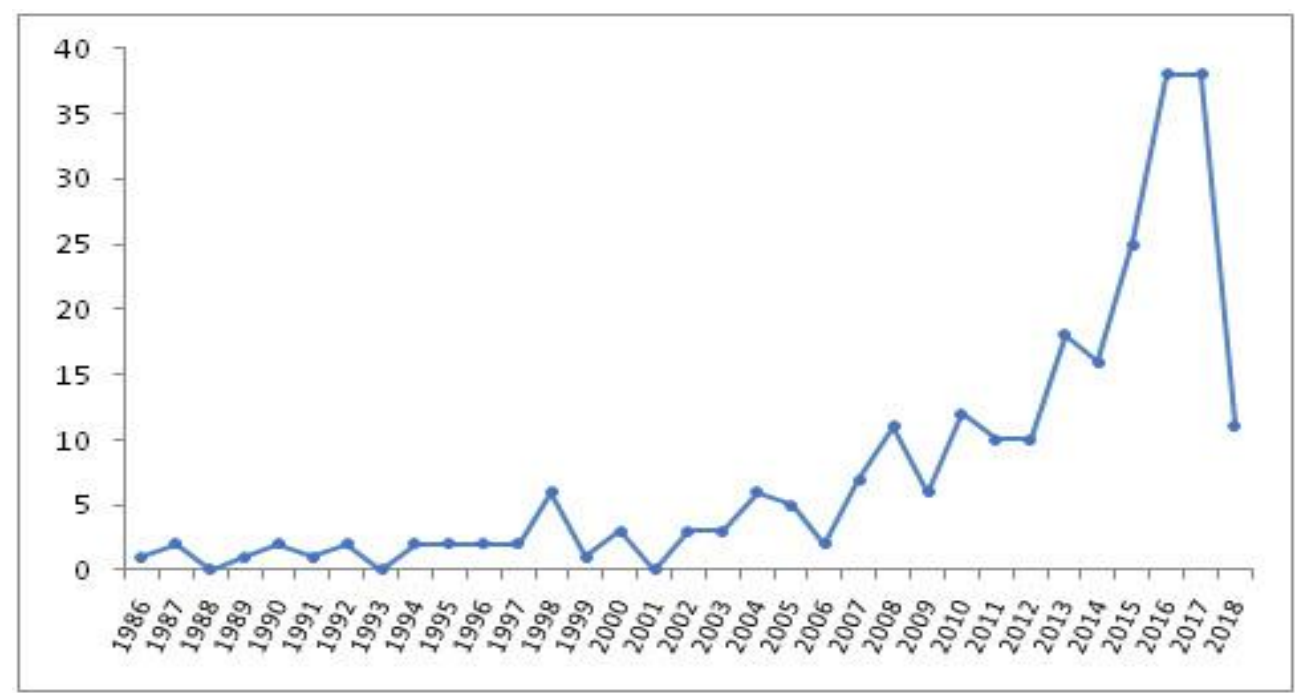

Figura 1. Número de tesis por año de lectura 


\subsection{Idiomas}

De acuerdo a los idiomas declarados en los registros de las distintas fuentes consultadas, la lengua más empleada en la redacción de las tesis es, con gran diferencia, el castellano con un total de 216 documentos $(87,1 \%)$. Se han empleado por parte de los doctorandos otras lenguas cooficiales en diversos territorios, aunque los trabajos en los que se han empleado estas suponen un porcentaje mucho menor $(10,08 \%)$ : catalán (22), gallego (2) y euskera (1). El catalán está presente casi desde el inicio del periodo estudiado (1989), mientras que el uso de las otras dos lenguas ha sido muy posterior (2006 y 2017, respectivamente). Se advierte una presencia meramente testimonial de algunas lenguas extranjeras como el inglés (5), el portugués (1) y el francés (1), aunque se aprecia un leve incremento de los documentos en inglés en los años 2016 y 2017.

\subsection{Universidades}

Tras normalizar las diferentes variantes encontradas sobre los nombres de las instituciones, se han podido identificar 48 universidades en las que se defendieron tesis de museología, de las 83 instituciones educativas que tienen registros incluidos en la base de datos TESEO. La incorporación de cada universidad a la lista de organismos productores de tesis ha resultado lenta, pues a mitad del periodo estudiado (2002) sólo 14 de las 48 entidades tenía un trabajo registrado.

En las 48 entidades se aprecia una cierta concentración de la producción. En 28 universidades las tesis defendidas han sido tres o menos, lo que representa un 17,74\% del total, mientras que en las 20 entidades restantes, incluidas en la tabla I, la producción alcanza un alto porcentaje $(82,26 \%)$. Entre las entidades más productivas destacan las cuatro siguientes, las cuales acumulan algo más de una tercera parte de la producción: Universidad Complutense de Madrid, Universitat de Barcelona, Universidad de Granada y Universitat Politècnica de València.

Los resultados de producción de las universidades se han considerado también de acuerdo a la ubicación de las instituciones por área geográfica y a su naturaleza jurídica. En cuanto a su localización, reseñar que Cataluña es la comunidad autónoma que tiene mayor número de universidades (9) y acumula mayor nivel de producción (56 tesis), seguida por la Comunidad de Madrid (7 universidades y 44 tesis), Andalucía (6 universidades y 40 tesis) y la Comunidad Valenciana (5 universidades y 30 tesis). El resto de comunidades autónomas reúnen menos cantidad de centros universitarios y sus niveles de producción son mucho menores. Si se atiende a la titularidad de los organismos, hay 41 universidades públicas y 5 privadas. Estas últimas suponen un 4,83\% de la producción de tesis frente al 95,17\% que han producido las entidades de carácter público. 
Tabla 1. Distribución de tesis doctorales por universidad ordenadas según cantidad producida $(\geq 4)$.

\begin{tabular}{|l|l|l|}
\hline Universidad & Frec. & Por. \\
\hline Universidad Complutense de Madrid & 26 & $10,48 \%$ \\
\hline Universitat de Barcelona & 25 & $10,08 \%$ \\
\hline Universidad de Granada & 17 & $6,85 \%$ \\
\hline Universitat Politècnica de València & 16 & $6,45 \%$ \\
\hline Universidad de Sevilla & 12 & $4,84 \%$ \\
\hline Universidad de Zaragoza & 10 & $4,03 \%$ \\
\hline Universitat de València & 10 & $4,03 \%$ \\
\hline Universitat Autònoma de Barcelona & 10 & $4,03 \%$ \\
\hline Universitat Politècnica de Catalunya & 9 & $3,63 \%$ \\
\hline Universidad de Murcia & 9 & $3,63 \%$ \\
\hline Universidad de Oviedo & 8 & $3,23 \%$ \\
\hline Universidad de Valladolid & 8 & $3,23 \%$ \\
\hline Universidad de Málaga & 7 & $2,82 \%$ \\
\hline Universidad Autónoma de Madrid & 7 & $2,82 \%$ \\
\hline Universidad de Salamanca & 6 & $2,42 \%$ \\
\hline Universidad de Santiago de Compostela & 6 & $2,42 \%$ \\
\hline Universidad de Navarra & 5 & $2,02 \%$ \\
\hline Universidad Nacional de Educación a Distancia & 5 & $2,02 \%$ \\
\hline Universidad Politécnica de Madrid & 4 & $1,61 \%$ \\
\hline Universidad de Deusto & 4 & $1,61 \%$ \\
\hline
\end{tabular}

En lo que se refiere a los departamentos universitarios, se han recogido 85 denominaciones diferentes en 207 registros de tesis en los que se han identificado de forma correcta. En el resto de los casos se ha comprobado que los registros no identifican el departamento o lo hace de forma errónea confundiéndolo con el nombre de la universidad.

Al recoger la producción bajo cada denominación se han obtenido unas cantidades que en la mayoría de los casos resultan muy bajas. Los departamentos más productivos son los de Historia del arte (19,3\%), Historia (3,86\%), Didáctica de las ciencias sociales $(3,36 \%)$ y Prehistoria y arqueología $(2,9 \%)$. Con el fin de facilitar la contabilidad de todos los registros con departamento identificado, se agruparon los elementos por las siguientes áreas de conocimiento que a continuación se relacionan por nivel de producción: Arte-Historia del arte $(28,5 \%)$, Ciencias de la Educación (23,67\%), Historia y Ciencias Historiográficas $(11,6 \%)$, Bellas Artes $(10,63 \%)$, Arquitectura $(8,21 \%)$, Ciencias de la Comunicación $(7,76 \%)$ y otras 
disciplinas (9,63\%), donde se agrupan departamentos de Filosofía, Psicología, Geografía, Sociología, Farmacia y Filología.

Si se consideran de forma conjunta tanto los datos de las universidades como de los departamentos, destacan por su productividad los grupos de departamentos pertenecientes a las siguientes universidades: Bellas Artes de la Universitat Politècnica de València (10), Ciencias de la Educación de la Universitat de Barcelona (9), Arquitectura de la Universitat Politècnica de Catalunya (8) e Historia del Arte de la Universidad de Granada (8).

\subsection{Análisis temático}

Para comprobar las materias que se han tratado en las tesis se ha recurrido a los descriptores del Tesauro de la UNESCO recogidos en los registros de la base de datos TESEO. En el conjunto total de registros de este estudio se han encontrado 9, fechados entre los años 2002 y 2004, que no incluyen descriptores, por lo que las cifras de resultados aquí expuestas se refieren a las 239 tesis con términos asignados y no a las 248 que se han obtenido en las consultas.

En las 239 tesis sobre las que se han especificado las materias se han contabilizado 635 descriptores, lo que deja una media de 2,65 descriptores por tesis. Por número de descriptores en cada registro los grupos más importantes son los correspondientes a descriptor único $(30,5 \%)$, tres $(26,78 \%)$ y cuatro $(26,36 \%)$. Es conveniente reseñar que desde el año 2005 en adelante no se ha recuperado ningún registro con más de cuatro descriptores.

En lo que se refiere al uso de cada descriptor se han identificado 137 diferentes, muchos de los cuales $(66,42 \%)$ se han usado dos veces o menos. Entre los descriptores más utilizados, recogidos en la tabla II, destaca la museología (138) y otros descriptores relativos a disciplinas como Arquitectura, Bellas Artes, Ciencias de la Comunicación, Ciencias de la Educación, Filosofía, Historia, Historia del Arte y Sociología.

Tabla 2. Descriptores empleados en indización de tesis por frecuencia de uso $(\geq 9)$

\begin{tabular}{|l|l|}
\hline Descriptor & Frec. \\
\hline Museología & 138 \\
\hline Historia del arte & 54 \\
\hline Historia & 23 \\
\hline Teoría, análisis y crítica de las bellas artes & 23 \\
\hline Ciencias de las artes y las letras & 16 \\
\hline Antropología cultural & 15 \\
\hline Historias especializadas & 15 \\
\hline Pedagogía & 14 \\
\hline Antropología & 12 \\
\hline Arquitectura & 12 \\
\hline Historia del arte de los siglos XIX y XX & 12 \\
\hline Política cultural & 11 \\
\hline
\end{tabular}




\begin{tabular}{|l|l|}
\hline Organización y planificación de la educación & 10 \\
\hline Pintura & 9 \\
\hline Sector de la educación & 9 \\
\hline
\end{tabular}

\subsection{Dirección de tesis}

En el corpus manejado se han identificado los directores en 244 tesis, quedando sin determinar dicha información en otras cuatro que se defendieron entre los años 1987 y 1990. En total se han contabilizado 280 directores, de los cuales 10 aparecen también en la lista de doctorandos. La producción de los directores se presenta muy distribuida: 243 de ellos (86,78\%) sólo han dirigido una tesis, mientras que sólo 29 $(10,36 \%)$ lo han hecho en dos ocasiones y $8(2,86 \%)$ han sido supervisores de tres o más tesis.

Por otra parte, en la tabla 3 sobre los directores más productivos, destaca una alta diversificación en la filiación institucional (siete universidades para ocho directores) y una gran concentración de tesis dirigidas en los últimos años del periodo estudiado.

$\mathrm{Si}$ se considera la presencia de directores por género se constata que hay un mayor número de hombres que de mujeres (152 frente a 128), aunque esa diferencia que se mantiene si se toma en consideración el género de los directores en base a su productividad. En ese caso las tesis dirigidas o codirigidas por hombres son $182 \mathrm{y}$ las dirigidas o codirigidas por mujeres son 153 . No obstante, la presencia de mujeres entre los directores más productivos es mayor que la de los hombres, como puede comprobarse en la tabla 3.

Tabla 3. Filiación institucional y productividad de directores con tres o más tesis.

\begin{tabular}{|l|l|l|l|}
\hline Director & Universidad & $\begin{array}{l}\text { Núm. } \\
\text { tesis }\end{array}$ & Años de lectura \\
\hline $\begin{array}{l}\text { Bellido Gant, María } \\
\text { Luisa }\end{array}$ & Granada & 6 & $\begin{array}{l}2013,2014,2015,2017 \\
(3)\end{array}$ \\
\hline Santacana Mestre, Joan & Barcelona & 5 & $\begin{array}{l}2005,2007,2010,2011, \\
2014\end{array}$ \\
\hline Asensio Brouard, Mikel & Autónoma de Madrid & 4 & $2005,2012,2013,2018$ \\
\hline Calaf Masachs, M. Roser & Oviedo & 4 & $2010,2014,2015,2017$ \\
\hline Fontal Merillas, Olaia & Valladolid & 4 & $2013,2014,2015,2018$ \\
\hline Lorente Lorente, J. Pedro & Zaragoza & 4 & $2014,2015,2016,2017$ \\
\hline Padró Puig, M. Carla & Barcelona & 4 & $2008,2009,2010,2013$ \\
\hline $\begin{array}{l}\text { Huerta Ramón, Ricard } \\
\text { V. }\end{array}$ & Valencia & 3 & $2013,2015,2016$ \\
\hline
\end{tabular}

La codirección se ha observado en 87 tesis $(32,25 \%)$, 80 de las mismas declaran dos codirectores y 7 reúnen hasta tres codirectores. Si se considera la producción por personas, se contabilizan que 162 directores $(57,1 \%)$ han codirigido tesis. Sin embargo, sólo 15 de estos han empleado más de una vez esta forma de colaboración durante el periodo analizado. Entre dichos directores destacan por su productividad 
en codirección los siguientes académicos: Mikel Asensio Brouard (4), Maria del Roser Calaf Masachs (3) y Olaia Fontal Merillas (3).

Respecto a las genealogías se han identificado 7 que muestran el mínimo de generaciones (dos) y 1 que recoge hasta tres generaciones, que aparecen todas recogidas en la figura 2. La genealogía más larga es también la más longeva (19862015) y está especialmente vinculada a la Universidad Complutense de Madrid. Dicha genealogía comienza con los catedráticos de Historia del Arte de la mencionada universidad, Joan Sureda Pons y Jesús Hernández Pereda, e incluyen en las dos primeras generaciones a sendos profesores de la UCM: Luis Alonso Fernández e Isabel García Fernández.

Entre las otras genealogías conviene destacar tres. Una es la iniciada por los catedráticos Federico Castro Morales y Fernando Martín Martín, que recoge hasta siete doctorandos en dos generaciones: la primera está formada por María Luisa Bellido Gant (Universidad de Granada) y la segunda por seis doctorandos dirigidos por dicha profesora. Otras dos genealogías destacables son las comenzadas por Joan Santacana Mestre (Universitat de Barcelona) en 2011 y 2014, en las que se recogen tesis sobre aspectos educativos de los museos.

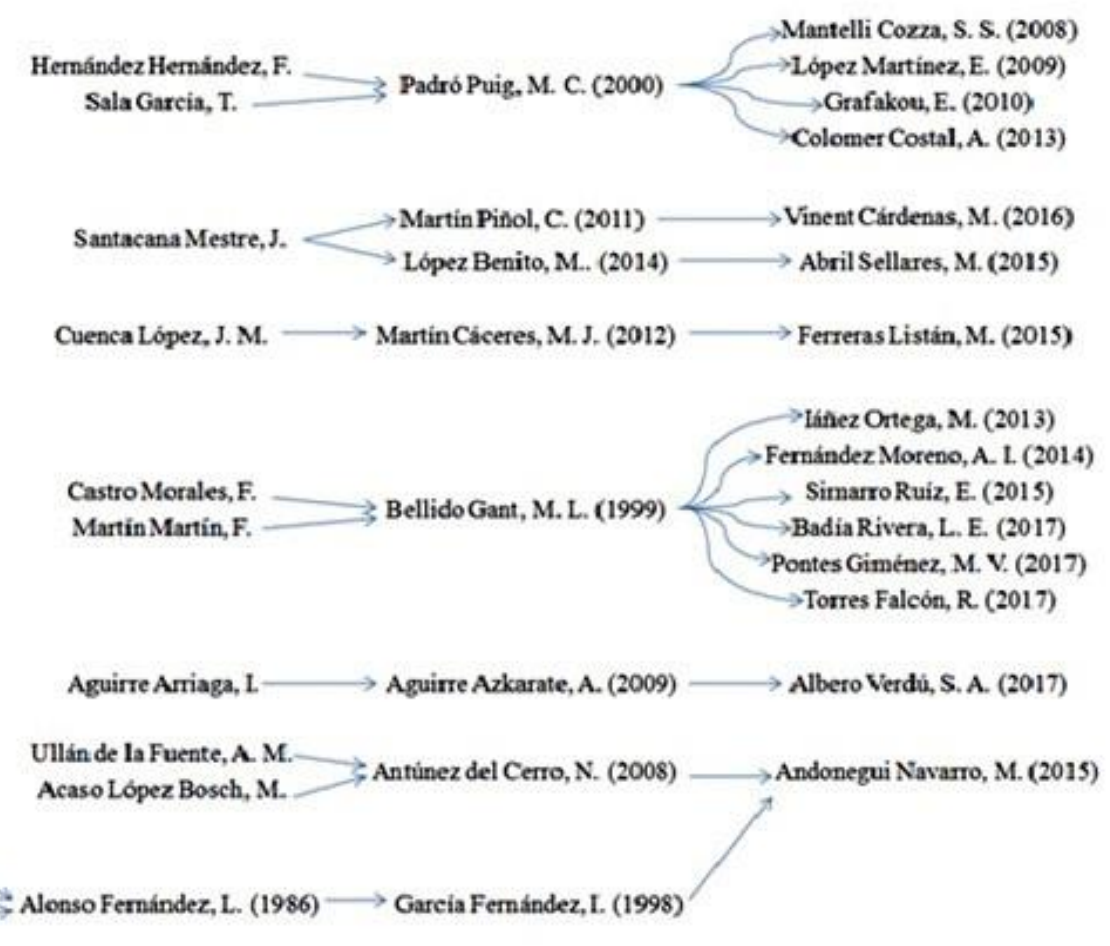

Figura 2. Genealogías académicas identificadas 


\subsection{Presencia en tribunales}

La información relativa a los miembros de los tribunales ha podido ser recuperada en casi todos los registros que se han manejado. Solamente en dos tesis faltan los nombres de los secretarios y uno de los vocales, por lo que se pierden los nombres de tres esos componentes. La cifra total de académicos que han formado parte de los tribunales evaluadores de tesis asciende a 801 , de los cuales $680(84,89 \%)$ solamente han participado en una ocasión, $71(8,86 \%)$ han estado en dos tribunales, $23(2,87 \%)$ han sido convocados tres veces y $27(3,37 \%)$ han intervenido en cuatro o más tribunales. En la tabla IV se incluyen información sobre los académicos que han tenido un mayor número de participaciones en tribunales de evaluación y los puestos que han desempeñado en los mismos.

$\mathrm{Al}$ atender la presencia en los tribunales por el género de los doctores, se constata que hay un porcentaje mayor de hombres $(62,8 \%)$ que de mujeres $(37,2 \%)$. La diferencia que se establece entre los géneros llega a ser incluso mayor para el caso de los presidentes de tribunales, entre los cuales la participación de las mujeres queda reducida a un poco más de la cuarta parte del total.

Tabla 4. Académicos con mayor número de participaciones en tribunales

\begin{tabular}{|l|l|l|l|l|l|}
\hline Nombre & Universidad & $\begin{array}{l}\text { Núm. } \\
\text { tribunales }\end{array}$ & Presidente & Secretario & Vocal \\
\hline $\begin{array}{l}\text { Calaf Masachs, María } \\
\text { Roser }\end{array}$ & Oviedo & 12 & 4 & 0 & 8 \\
\hline Prats Cuevas, Joaquin & Barcelona & 9 & 5 & 2 & 2 \\
\hline Diaz Balerdi, Ignacio & País Vasco & 8 & 0 & 2 & 6 \\
\hline $\begin{array}{l}\text { Hernández Cardona, F. } \\
\text { Xavier }\end{array}$ & Barcelona & 8 & 6 & 2 & 0 \\
\hline $\begin{array}{l}\text { Huerta Ramon, Ricard } \\
\text { Vicent }\end{array}$ & Valencia & 8 & 0 & 2 & 6 \\
\hline Santacana Mestre, Joan & Barcelona & 8 & 4 & 2 & 2 \\
\hline Henares Cuéllar, Ignacio & Granada & 7 & 6 & 0 & 1 \\
\hline Fontal Merillas, Olaia & Valladolid & 7 & 0 & 0 & 7 \\
\hline $\begin{array}{l}\text { Calle de la Calle, Román } \\
\text { de la }\end{array}$ & Valencia & 6 & 3 & 0 & 3 \\
\hline $\begin{array}{l}\text { Cuenca López, José } \\
\text { María }\end{array}$ & Huelva & 6 & 1 & 2 & 3 \\
\hline $\begin{array}{l}\text { Hernández Hernández, } \\
\text { Francisca }\end{array}$ & $\begin{array}{l}\text { Complutense y } \\
\text { Autónoma de }\end{array}$ & 5 & 1 & 0 & 4 \\
\hline $\begin{array}{l}\text { Juanola Tarradellas, } \\
\text { Roser }\end{array}$ & Girona & 5 & 4 & 0 & 1 \\
\hline $\begin{array}{l}\text { Rivero Gracia, María } \\
\text { Pilar }\end{array}$ & Zaragoza & 5 & 1 & 0 & 4 \\
\hline Rodríguez Ortega, Nuria & Málaga & 5 & 0 & 1 & 4 \\
\hline $\begin{array}{l}\text { Hernández Hernández, } \\
\text { Fernando }\end{array}$ & Barcelona & 4 & 3 & 0 & 1 \\
\hline
\end{tabular}




\begin{tabular}{|l|l|l|l|l|l|}
\hline Parralo Dorado, Manuel & Complutense & 4 & 4 & 0 & 0 \\
\hline $\begin{array}{l}\text { Pita Andrade, José } \\
\text { Manuel }\end{array}$ & $\begin{array}{l}\text { Complutense y } \\
\text { Granada }\end{array}$ & 4 & 4 & 0 & 0 \\
\hline
\end{tabular}

Entre los académicos con mayor presencia de la tabla IV se distingue a María del Roser Calaf Masachs, Joaquín Prats Cuevas, Francesc Xavier Hernández Cardona y Ignacio Henares Cuellar, tanto por el número de tribunales en los que han participado como por las veces en las que han ejercido la presidencia.

Conviene también señalar entre los académicos la presencia de los catedráticos Manuel Parralo Dorado y José Manuel Pita Andrade, quienes demuestran un elevado prestigio entre la comunidad académica ya que siempre que han formado parte de un tribunal lo han hecho ocupando la presidencia.

Entre las instituciones de los académicos con mayor índice de participación en tribunales destaca la Universitat de Barcelona. También se ha identificado un importante número de miembros de tribunales (3) pertenecientes a la Universidad Complutense. Sin embargo, dos de ellos estuvieron también en otras instituciones durante el periodo sometido a revisión, lo que reduce la cifra de tribunales. En el caso de José Manuel Pita Andrade actuó como catedrático de la Universidad Complutense solamente en el primer tribunal que presidió (1986). Tras su jubilación en 1987, ejerció durante algún tiempo como profesor emérito de la Universidad de Granada, época en la que fue presidente de otros dos tribunales evaluadores, ambos reunidos en el año 1998.

\section{Conclusiones}

El presente estudio de tesis sobre museología pone de manifiesto el interés que han demostrado por dicha materia los investigadores en formación, procedentes de diversas disciplinas científicas, de las universidades españolas, con una alta distribución de centros productores y del personal encargado de la dirección y evaluación de las tesis.

La producción global de tesis ha ido incrementándose de manera desigual hasta el año 2006, momento en el que comienza a ascender de manera abrupta, aunque con algunos altibajos. Este incremento de la producción de tesis doctorales en los años más recientes demuestra la consolidación de la museología como materia de investigación en las universidades españolas. Dicha materia debe considerarse una ciencia multidisciplinar en la que se abordan estudios desde diferentes enfoques, como puede deducirse de los descriptores utilizados para describir el contenido de las tesis doctorales.

En lo relativo a las instituciones de lectura se puede concluir que se está dando una tendencia a la distribución por universidades en los años más recientes frente a la concentración que existía durante el primer periodo. A pesar de esa tendencia a la distribución observada en la producción de tesis, en dieciséis universidades catalanas y madrileñas se ha defendido un porcentaje superior al cuarenta por ciento de las 
tesis sobre museología defendidas en España, lo que demuestra que la distribución es algo menor si se considera desde un punto de vista geográfico.

Entre los académicos se ha constatado un alto grado de transitoriedad, entendida esta como la cantidad de personas que realizan una actividad en una única ocasión, y que muchas tesis han sido codirigidas por dos doctores. De ello se infiere que existe un importante nivel de colaboración entre académicos. En lo que se refiere a las genealogías entre directores y doctorandos, es importante señalar que apenas han empezado a conformarse las mismas en los años más recientes del periodo observado, por lo que la mayoría presentan el mínimo número de generaciones posibles.

En los miembros de los tribunales se ha dado también un elevado número de académicos con una escasa participación, quedando solamente 27 con un elevado número de intervención en tribunales, lo que es indicio de su prestigio entre los docentes dedicados a la ciencia de los museos.

También es importante señalar la desigualdad entre géneros en los conjuntos de personas estudiados. En el grupo de doctorandos las mujeres representan hasta dos terceras partes del total. Sin embargo, sus porcentajes como directoras de tesis o miembros de tribunales son mucho menores (45,8\% y 37,2\%, respectivamente). En base a esos datos se puede concluir que las mujeres, como doctoras, representan un importante grupo en la investigación sobre museologia, pero que dicha importancia no se ve reflejada posteriormente en puestos de dirección o evaluación académica, donde los hombres son mayoría.

A modo de conclusión general, se puede afirmar que este novedoso estudio bibliométrico de las tesis doctorales sobre museología en universidades españolas ha servido para conocer la productividad de las diferentes instituciones y la actividad de dirección y evaluación de los principales académicos.

\section{Referencias bibliográficas}

Beladrón Pazo, A. J.; Manchado Pérez, B.; Correyero Ruiz, B. (2019). La investigación sobre publicidad en la universidad española. Características y temáticas de las tesis doctorales (1976-2016). Revista Latina de Comunicación Social, 74, 767-785. http://dx.doi.org/10.4185/RLCS-2019-1356-39. < http://www.revistalatinacs.org/074pap er/1356/39es.html>

Bovolenta Ovigli, D. (2015). Panorama das pesquisas brasileiras sobre educação em museus de ciências. Revista Brasileira de Estudos Pedagógicos, 96 (244), 577-595. http://dx.doi.org/10.1590/S2176-6681/33891329. < <ttps://www.scielo.br/pdf/rbeped/v9 6n244/2176-6681-rbeped-96-244-00577.pdf>

Casanueva Roche, C.; Escobar Pérez, B.; Larrinaga González, C. (2007). Red social de Contabilidad en España a partir de los tribunales de tesis. Revista Española de Financiación y Contabilidad, 37 (136), 707-722.

Castelló-Cogollos, L.; Bueno Cañigral, F. J.; Valderrama Zurián, J. C. (2019). Análisis de redes sociales y bibliométrico de las tesis españolas sobre drogodependencias en la base de datos TESEO. Adicciones, 31 (4), p. 309-323. http://dx.doi.org/10.20882/adicciones. 1150. <http://www.adicciones.es/index. php/adicc iones/article/view/1150/1010> 
Curiel-Martín, E.; Fernández-Cano, A. (2015). Análisis Cienciométrico de Tesis Doctorales Españolas en Didáctica de las Ciencias Sociales (1976-2012). Revista Española de Documentación Científica, 38 (4): e110. http://dx.doi.org/10.3989/ redc.2015.4.1282. <http://redc.revistas.csic.es/index.php/redc/article/viewFile/913/1308>

Delgado López-Cózar, E.; Torres-Salinas, D.; Jiménez-Contreras, E.; Ruiz-Pérez, R. (2006). Análisis bibliométrico y de redes sociales aplicado a las tesis bibliométricas defendidas en España (1976-2002): temas, escuelas científicas y redes académicas. Revista Española de Documentación Científica, 29 (4), 493-524. https://doi.org/10.3989/redc.2006.v29.i4. 306. <http://redc.revistas.csic.es/ index.php/redc/article/view/306/381>

Díaz Campo, J. (2014). La investigación sobre Internet en las facultades de Comunicación españolas. Análisis bibliométrico de tesis doctorales (1997-2012). Documentación de las Ciencias de la Información, 37, 305-320. http://dx.doi.org/10.5209/rev_DCIN.2014.v37. 46828. https://revistas.ucm.es/index.php/DCIN/article/view/46828/43940>

Díaz Campo, J. (2015). Análisis bibliométrico de las tesis doctorales sobre ética de los medios de comunicación presentadas en España (1979-2013). Doxa Comunicación, 20, 65-88. https://doi.org/10.31921/doxacom.n20a3. <https://repositorioinstitucional.ceu.es/ bitstream/10637/7293/1/Analisis_JesusDiaz_Doxa_2015.pdf>

Fuentes Pujol, E.; Argimbau Vivó, L. (2010). Las tesis doctorales en España (1997-2008): análisis, estadísticas y repositorios cooperativos. Revista Española de Documentación Científica, vol. 33 (1), 63-89. 10.3989/redc.2010.1.711. <http://redc.revistas.csic.es/ index.php/redc/article/view/526/584>

García Rubio, A.; Gómez García, C. I.; Martínez Clares, P.; Ruíz García, M. J. (2019). Análisis bibliométrico de tesis doctorales españolas en lactancia materna, entre los años 1980-2015. Cultura de los Cuidados, 23 (54). http://dx.doi.org/10.14198/cuid.2019.54.16

Jiménez-Contreras, E., Ruiz-Pérez, R.; Delgado López-Cózar, E. (2014). El análisis de las tesis doctorales como indicador evaluativo: reflexiones y propuestas. Revista de Investigación Educativa, 32 (2), 295-308. https://doi.org/10.6018/ rie.32.2.197401. <https://revistas.um.es/rie/article/view/197401/163181>

Machan, K.; Sendra Portero, F. (2018). Las tesis doctorales en radiodiagnóstico: estudio de la producción española entre 1976 y 2011. Radiología, 60 (5), 394-403. DOI: 10.1016/j.rx.2018.03.005

Maz-Machado, A.; Bracho-López, R.; Torralbo-Rodríguez, M.; Gutiérrez-Arenas, M. P.; Jiménez-Fanjul, N.; Adamuz-Povedano, N. (2012). Redes académicas generadas por las tesis doctorales de educación matemática en España. Revista de Investigación Educativa, 30 (2), 271-286. <https://revistas.um.es/rie/article/ view/116421/148841>

Marcos Recio, J. C.; Martínez Pestaña, M. J.; Blasco López, M. F. (2012). Producción y dirección de tesis doctorales sobre publicidad en la universidad española (1971-2010). Revista Española de Documentación Científica, 35 (3), 433-452. https://doi.org/10.3989/redc.2012.3.890 <http://redc.revistas.csic.es/index.php/redc/arti cle/view/751>

Moreno-Fernández, O.; Moreno-Crespo, P. (2016). Análisis bibliométrico de las tesis doctorales españolas indexadas con el descriptor "Sector de la educación" (1976/2014). Revista Española de Documentación Científica, 39 (3): e146. http://dx.doi.org/10.3989/redc.2016.3.1331.

<http://redc.revistas.csic.es/index.php/redc/article/view/950/1416>

Olivera Zaldua, M.; Sánchez Vigil, J. M.; Marcos Recio, J. C. (2016). Análisis de las tesis doctorales sobre fotografía en la universidad española (enero de 2013-marzo de 2016). Ibersid: Revista De Sistemas de Información y Documentación, 10 (2), 13-20. < https://www.ibersid.eu/ojs/index.php/ibersid/article/view/4328> 
Osca-Lluch, J.; Haba, J.; Fonseca, S.; Civera, C.; Tortosa, F. (2013). Tesis doctorales española sobre análisis bibliométrico en psicología. Aula Abierta, 41 (2), 99-110. <https://redined.mecd.gob.es/xmlui/bitstream/handle/11162/97230/Aula\%20abierta_Vo 1.41_n.2_p99-110.pdf?sequence=1\&isAllowed=y $>$

Ramos-Pardo F. J.; Sánchez-Antolín, P. (2017). Production of educational theory doctoral theses in Spain (2001-2015). Scientometrics, 112 (3), 1615-1630. https://doi.org/10.10 07/s11192-017-2435-6

Repiso, R., Torres, D.; Delgado, E. (2011). Análisis bibliométrico y de redes sociales en tesis doctorales españolas sobre televisión (1976/2007). Comunicar, 19 (37), 151-159. https://doi.org/10.3916/C37-2011-03-07.

$<$ https://www.revistacomunicar.com/index.php?contenido=detalles\&numero=37\&articu lo=37-2011-18>

Repiso Caballero, R., Torres Salinas, D.; Delgado López-Cózar, E. (2011). Análisis de la investigación sobre Radio en España: una aproximación a través del Análisis Bibliométrico y de Redes Sociales de las tesis doctorales defendidas en España entre 1976-2008. Estudios Sobre El Mensaje Periodístico, 17 (2), 417-429. https://doi.org/10.5209/rev_ESMP.2011.v17.n2.38123.

<https://revistas.ucm.es/index.php/ESMP/article/view/38123/36875>

Repiso, R.; Torres Salinas, D.; Delgado López-Cózar, E. (2013). La investigación científica sobre Cine en España a partir de sus tesis doctorales: Análisis de redes sociales (19782007). Icono 14, vol. 11 (2), 385-404. https://doi.org/10.7195/ri14.v11i2.530. <https://icono14.net/ojs/index.php/icono14/article/view/530/431>

Weis, H. (dir.). (1989). La muséologie selon Georges Henri Rivière, Paris, Dunod.

Sánchez-Vigil, J. M.; Marcos Recio, J. C.; Olivera-Zaldua, M. (2016). Tesis doctorales sobre fotografía en la universidad española. Análisis de la producción y dirección (1976-2012). Revista Española de Documentación Científica, 37 (1): e034. http://dx.doi.org/10.3989/redc.2014.1.1073. http://redc.revistas.csic.es/index.php/redc/article/view/837/1074>

Sanchidrian Blanco, C. (2016). Tesis de Historia de la Educación en la base de datos TESEO (España, 2000-2010). Espacio, Tiempo y Educación, 3 (1), 273-292. http://dx.doi.org/10.14516/ete.2016.003.001.14

Sardo Menezes, G.; Barbosa Vianna, W.; Matias, M. (2019). O uso de Realidade Aumentada no contexto dos museus: o portfólio brasileiro de teses e dissertações até 2017 . Em questão, 25 (3), 246-268. http://dx.doi.org/10.19132/1808-5245253.246-2628. <https://seer.ufrgs.br/EmQuestao/article/view/86096/53011>

Segarra-Saavedra, J.; Villena-Alarcón, E. (2020). Análisis bibliométrico, de redes sociales y citas en Google académico de las tesis doctorales defendidas en España sobre adicciones (1976-2018). Health and Adicctions, 20 (2), 170-181. https://doi.org/10.21134/haaj.v20i2.551. <https://ojs.haaj.org/index.php?journal= haaj\&page $=$ article \&op $=$ view $\&$ path $\% 5 \mathrm{~B} \% 5 \mathrm{D}=551>$

TESEO (2020). <https://www.educacion.gob.es/teseo/irGestionarConsulta.do>. [Consulta:10/04/2020]. 